\title{
Vitexin protects dopaminergic neurons in MPTP-induced Parkinson's disease through PI3K/Akt signaling pathway
}

This article was published in the following Dove Press journal:

Drug Design, Development and Therapy

\section{Ming $\mathrm{Hu}$ \\ Fangming $\mathrm{Li}$ \\ Weidong Wang}

Department of Neurology, Shenzhen Hospital, Southern Medical University, Shenzhen, Guangdong, China
Correspondence: Ming Hu Department of Neurology, Shenzhen Hospital, Southern Medical University, I 333 Xinhu Road, Shenzhen, Bao'an District, Guangdong Province 518100 , China

Tel +8675523360593

Email huminggdsz@163.com

\begin{abstract}
Parkinson's disease (PD) is a progressive neurodegenerative disease which is characterized by the degeneration of dopaminergic neurons in the substantia nigra pars compacta (SNpc).
\end{abstract}

Methods: In this study, the neuroprotective effect of vitexin (Vit), a flavonoid compound isolated from Crataegus pinnatifida Bunge was examined in PD models both in vitro and in vivo.

Results: On SH-SY5Y cells, methyl-4-phenylpyridine (MPP ${ }^{+}$) treatment suppressed cell viability, induced apoptosis, and increased Bax/Bcl-2 ratio and caspase-3 activity. However, Vit improved these parameters induced by $\mathrm{MPP}^{+}$treatment significantly. Further study disclosed that Vit enhanced the phosphorylation of PI3K and Akt which was downregulated by MPP ${ }^{+}$in SH-SY5Y cells, the effect of which could be blocked by PI3K inhibitor LY294002 and activated by PI3K activator IGF-1. Moreover, results from the pole test and traction test suggested that Vit pretreatment prevented bradykinesia and alleviated the initial lesions caused by 1-methyl-4-phenyl-1,2,3,6tetrahydropyridine (MPTP) in MPTP-treated mouse PD model. Vit also enhanced the activation of $\mathrm{PI} 3 \mathrm{~K}$ and Akt and suppressed the ratio of Bax/Bcl-2 and caspase-3 activity in MPTP-treated mice.

Conclusion: Taken together, this study demonstrated that Vit protected dopaminergic neurons against $\mathrm{MPP}^{+} / \mathrm{MPTP}$-induced neurotoxicity through the activation of PI3K/Akt signaling pathway. Our findings may facilitate the clinical application of Vit in the therapy of PD.

Keywords: vitexin, MPTP, Parkinson's disease, PI3K/Akt, neuroprotective

\section{Introduction}

Parkinson's disease (PD) is a progressive neurodegenerative disease which is characterized by the degeneration of dopaminergic neurons in the substantia nigra pars compacta $(\mathrm{SNpc})$ and a significant reduction of striatal dopamine primarily among the old people around the world. ${ }^{1}$ Although the exact pathogenesis of PD remains limited, a great number of studies have revealed that excessive generation of reactive oxygen species (ROS), oxidative stress and mitochondrial dysfunction contribute to the pathogenesis of PD. ${ }^{2-4}$ Nowadays, the main drug treatment for PD is the supplement of dopamine by L-dopa. However, dopamine supplement only relieves the symptoms of the disease instead of slowing down the progression of PD, and long-term use of L-dopa can lead to disabling fluctuations and dyskinesias. ${ }^{5}$ Therefore, finding a new drug against PD which can alleviate the symptoms of PD as well as prevent neurodegeneration and restore the degenerated dopaminergic neurons is important for the treatment of PD.

The neurotoxin 1-methyl-4-phenyl-1,2,3,6-tetrahydropyridine (MPTP), which is a well-known mitochondrial complex I inhibitor, can selectively damage dopaminergic neurons in SNpc. After crossing the blood-brain barrier, MPTP is converted to its 
active metabolite methyl-4-phenylpyridine $\left(\mathrm{MPP}^{+}\right)$, which can impair mitochondrial energy metabolism, induce ROS, and finally result in the death of dopaminergic neurons. Therefore, $\mathrm{MPP}^{+}$or MPTP is often used to construct PD model in vitro or in vivo. ${ }^{6-8}$

Recently, the neuroprotective role of flavonoids has received much attention from researchers. Flavonoid is reported to exert a multiplicity of neuroprotective actions in brain including protecting neurons against injury induced by neurotoxins, suppressing neuroinflammation, and promoting memory through specific interactions within the ERK and PI3K/AKT signaling pathways. ${ }^{9,10}$ Vitexin (Vit), a flavonoid compounds, is isolated from the leaf of Crataegus pinnatifida Bunge. ${ }^{11}$ It has been discovered to exert antiinflammatory, anti-oxidative, anti-tumor, and neuroprotective effects. ${ }^{12-15}$ However, little is known whether Vit could benefit PD therapy.

In the present study, we hypothesized that Vit protected dopaminergic neurons and alleviated PD through modulating $\mathrm{PI} 3 \mathrm{~K} / \mathrm{AKT}$ signaling pathway. In order to verify this hypothesis, the anti-PD effect of Vit was evaluated in MPTPinduced mice and $\mathrm{MPP}^{+}$-treated dopaminergic neuronal cell line, SH-SY5Y. We observed that Vit treatment protected dopaminergic neurons from the injury of MPTP or $\mathrm{MPP}^{+}$ through activating the PI3K/AKT signaling pathway. Our finding may contribute to the application of Vit for the treatment of PD.

\section{Materials and methods}

\section{Chemical compounds and reagents}

Vit (chemical structure is shown in Figure 1) obtained from Selleck Inc (Westlake Village, CA, USA), was dissolved

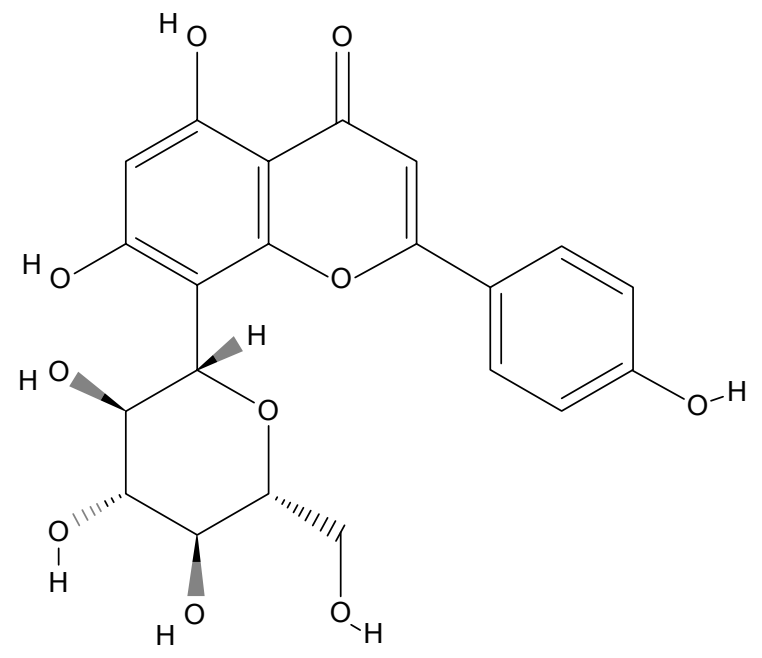

Figure I The chemical structure of vitexin. by DMSO (Sigma-Aldrich Co., St Louis, MO, USA) and stored at $-20^{\circ} \mathrm{C}$. $\mathrm{MPP}^{+}$iodide (D048-100MG) and MPTP (SLBB6890V) were purchased from Sigma-Aldrich Co. MTT, DAPI, and the Annexin V-FITC/PI Staining Apoptosis Detection Kit were also purchased from Sigma-Aldrich Co. Antibodies against Bax, Bcl-2, phospho-PI3K (p-PI3K), PI3K, phospho-Akt (p-Akt), Akt, and $\beta$-actin were purchased from Cell Signaling Technology (Danvers, MA, USA). Madopar was purchased from Hoffman-La Roche Ltd. (Basel, Switzerland). LY294002, a PI3K inhibitor, and PI3K activator IGF-1were purchased from Selleckchem (Boston, MA, USA). Caspase-3 Activity Assay Kit was purchased from EMD Millipore (Billerica, MA, USA).

\section{Cell culture and treatment}

Human neuroblastoma SH-SY5Y cells were purchased from American Type Culture Collection (ATCC) and cultured in DMEM/F12 medium (Thermo Fisher Scientific, Waltham, MA, USA) supplemented with $10 \%$ fetal bovine serum (FBS) (HyClone; GE Healthcare Life Science, Logan, UT, USA) at $37^{\circ} \mathrm{C}$ with $5 \% \mathrm{CO}_{2}$. After pretreated with various concentrations of Vit $(0,10,20$, and $40 \mu \mathrm{M})$ for 2 hours, some of the cells were incubated with $1 \mathrm{mM} \mathrm{MPP}^{+}$for 24 hours. Some other SH-SY5Y cells were treated with only various concentrations of Vit $(0,10,20,40$, or $100 \mu \mathrm{M})$ for 2 hours. LY294002 was used to treat cells for 24 hours at the concentration of $10 \mu \mathrm{M}$, and IGF-1 was used to treat cells for 24 hours at the concentration of $50 \mathrm{ng} / \mathrm{mL}$.

\section{Cell viability assay}

Cell viability was detected through MTT assay. After treated with Vit or $\mathrm{MPP}^{+}$, different groups of cells were seeded into 96-well plates at a density of $5 \times 10^{5}$ cells $/ \mathrm{mL}$. Then, $10 \mu \mathrm{L}$ of MTT solution was added in each well and incubated for 4 hours. After that, the medium was removed and the formazan crystals formed in the viable cells were dissolved by adding $150 \mu \mathrm{L}$ of DMSO. The absorbance of the solution at $560 \mathrm{~nm}$ was measured by a microplate reader (Bio-Rad Laboratories Inc., Hercules, CA, USA).

\section{Cell apoptosis assay}

Annexin V-FITC/PI Staining Apoptosis Detection Kit was used for cell apoptosis assay. Different groups of cells were collected and washed with ice-cold PBS. Then $3 \times 10^{5}$ of cells were resuspended and incubated with $5 \mu \mathrm{L}$ of Annexin V-FIFC and $10 \mu \mathrm{L}$ of PI. Cell apoptosis was analyzed by a flow cytometer (BD Biosciences, San Jose, CA, USA). 


\section{Western blot analysis}

Proteins were extracted from cells or substantia nigra (SN) tissues after SN dissection by using radioimmunoprecipitation assay (RIPA) lysis buffer (Beyotime Institute of Biotechnology, Shanghai, China) according to the manufacturer's protocol. The same amount of proteins from each sample were separated by $10 \%$ SDS-PAGE gel and then transferred into PVDF membranes (EMD Millipore). After blocking with $5 \%$ non-fat milk solution at room temperature for 1 hour, the membranes were incubated with respective primary antibodies overnight at $4^{\circ} \mathrm{C}$. Then, the membranes were incubated with respective secondary antibodies. ECL system (Bio-Rad Laboratories Inc.) was used for the detection of antibodybound proteins according to manufacturer's instructions.

\section{Determination of caspase- 3 activity}

Cells or brain tissues were homogenized via centrifugation at $12,000 \times g$ for 10 minutes at $4^{\circ} \mathrm{C}$. The reaction buffer containing $10 \mathrm{mM}$ dithiothreitol was added to the supernatants of each sample, and then DEVD-AFC substrate was added and incubated for 2 hours at $37^{\circ} \mathrm{C}$. Fluorescence intensity was detected at $360 \mathrm{~nm}$ excitation and $450 \mathrm{~nm}$ emission using a fluorescence microplate reader.

\section{Animals and treatment}

All animal experiments complied with the National Institutes of Health (USA) guide for the care and use of Laboratory animals (NIH Publications No 8023, revised 1978) and were approved by the Medical Ethics Committee of Shenzhen Hospital of Southern Medical University (China). Male C57BL/6 mice, 12-week old, were provided by the Animal Center of Shenzhen Hospital of Southern Medical University. Animals were housed at an ambient temperature of $23^{\circ} \mathrm{C} \pm 1^{\circ} \mathrm{C}$ and relative humidity $60 \% \pm 10 \%$ under a 12 -hour light/ dark cycle and were allowed free access to water and food. A total of 32 mice were randomly divided into four groups with eight mice in each group: control group, MPTP group, MPTP + Vit group, MPTP + Madopar group. Madopar which contains levodopa and benserazide and often used for PD treatment was served as a positive control drug to assess the relative therapeutic efficacy of Vit in our experiments. Mice in MPTP + Vit group and MPTP + Madopar group received oral administration of Vit or Madopar at $50 \mathrm{mg} / \mathrm{kg}$ daily, while mice in the other groups received the same volume of saline daily. MPTP $(30 \mathrm{mg} / \mathrm{kg} / \mathrm{d})$ was given intraperitoneally to mice in MPTP, MPTP + Vit, and MPTP + Madopar groups for 5 consecutive days 1 hour post-Vit or postMadopar administration from day 11 to day 15 (Figure 2).

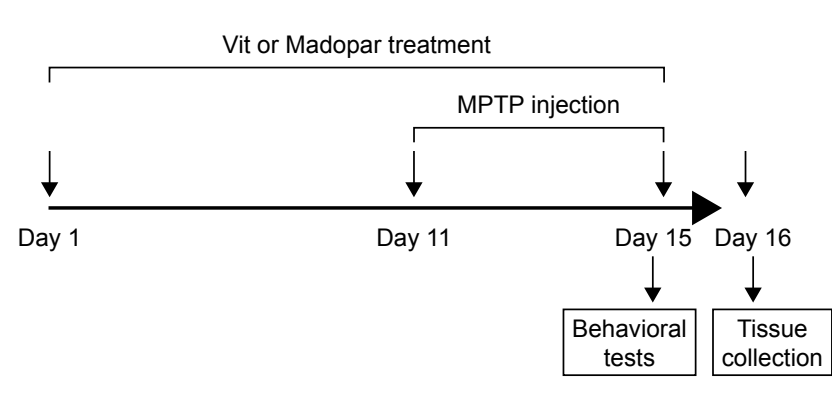

Figure 2 Timeline for the entire experiment.

Abbreviations: Vit, vitexin; MPTP, I-methyl-4-phenyl-I,2,3,6-tetrahydropyridine.

After performing the pole test and traction test on the fifteenth day, mice were sacrificed and the brains were collected for Western blot and determination of caspase-3 activity.

\section{Behavioral test}

Pole test is a useful method to evaluate bradykinesia in mouse PD models. We performed the pole test on the fifteenth day after the last MPTP injection. Mice were held on the top of a pole (diameter $10 \mathrm{~mm}$, height $52 \mathrm{~cm}$, with a rough surface). The time taken by the mice to turn down completely was recorded as the T-turn (time to turn).

The traction test is used to measure muscle strength and equilibrium, and it was also performed on the fifteenth day after the last MPTP injection. The mouse forepaws were placed on a rope (diameter $5 \mathrm{~mm}$ ), while its hind limb placements were scored from 1 to 3 , with the lowest score indicating the most severe deficit. The score was assessed according to the following criteria: if both hind limbs seized the rope, the score was 3 and if one or no hind limb seized the rope, the score was 2 or 1, respectively. In addition, the mice were allowed to hang upside down. Animals were allowed to stay on the rope for 30 seconds and the falling time was recorded.

\section{Statistical analysis}

All results were presented as mean \pm standard deviation (SD). Student's $t$-test was performed to analyze the difference between groups. Statistical analysis was performed with SPSS software (version 19). Values of $P<0.05$ were considered statistically significant.

\section{Results \\ Vit protects $\mathrm{SH}-\mathrm{SY} 5 Y$ cells against $\mathrm{MPP}^{+}$-induced toxicity and apoptosis}

Treatment with $\mathrm{MPP}^{+}$was shown to result in $>50 \%$ reduction of cell viability of SH-SY5Y cells. However, treatment with 

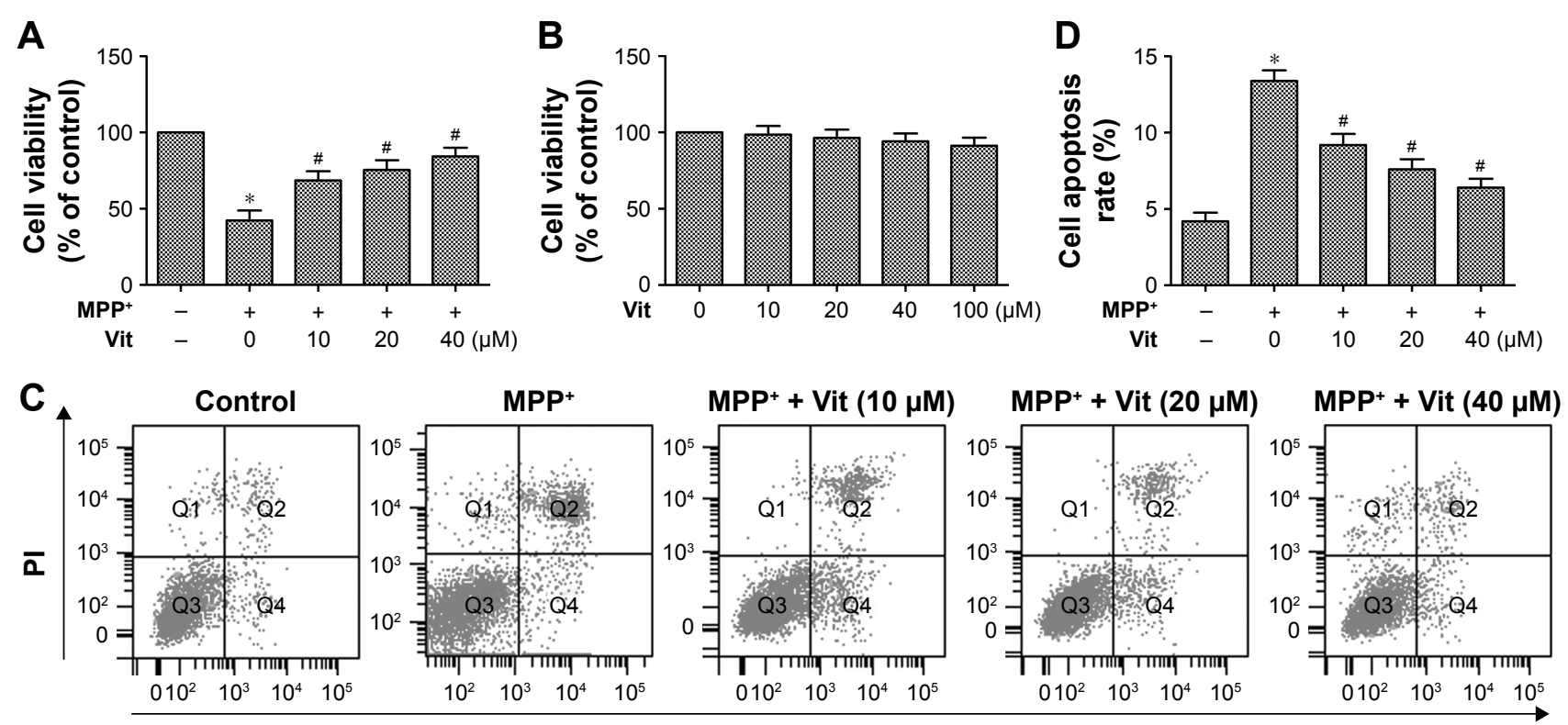

E

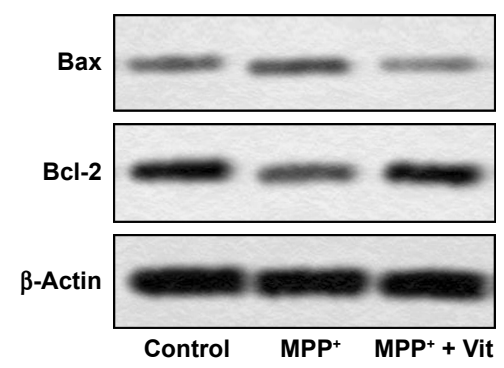

$\mathbf{F}$

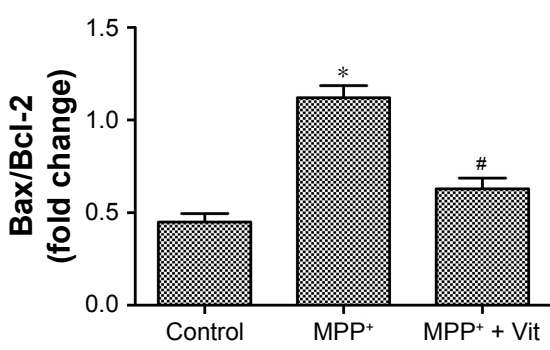

G

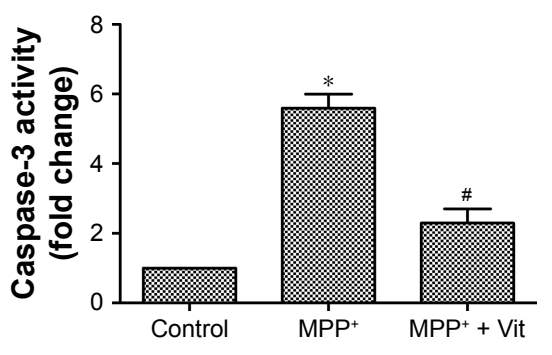

Figure 3 Vitexin protects SH-SY5Y cells against MPP+-induced toxicity and apoptosis. SH-SY5Y cells received different concentrations of Vit treatment in the presence or absence of MPP+. (A and B) Cell viability was detected through MTT. (C and D) Cell apoptosis rate was detected through flow cytometry. (E and F) Relative expression of Bax and $\mathrm{Bcl}-2$ was detected through Western blot. (G) Caspase-3 activity was measured using Caspase-3 Activity Assay Kit. The bars showed mean \pm SD of three independent experiments. ${ }^{* P}<0.05$ compared with control group, ${ }^{*} P<0.05$ compared with MPP ${ }^{+}$-treated group.

Abbreviations: Vit, vitexin; MPP, methyl-4-phenylpyridine.

Vit rescued this reduction significantly in a dose-dependent manner (Figure $3 \mathrm{~A},{ }^{*} P<0.05,{ }^{*} P<0.05$ ). In order to examine whether Vit could affect cell proliferation of SH-SY5Y cells, the cells were treated with different concentrations of Vit without $\mathrm{MPP}^{+}$. The data indicated that Vit ranging from 10 to $100 \mu \mathrm{M}$ did not have significant influence on cell viability (Figure 3B). Moreover, $\mathrm{MPP}^{+}$treatment increased cell apoptosis rate remarkably while $\mathrm{Vit}$ treatment reduced this increased cell apoptosis rate dose-dependently (Figure 3C and $\left.\mathrm{D},{ }^{*} P<0.05,{ }^{\#} P<0.05\right)$. To confirm the effect of Vit on $\mathrm{MPP}^{+}$-induced cell apoptosis, several apoptosis-related molecules were investigated. We observed that $\mathrm{MPP}^{+}$treatment increased caspase-3 activity and the expression of Bax while decreased the expression of Bcl-2, thus leading to the upregulation of $\mathrm{Bax} / \mathrm{Bcl}-2$ ratio. When pretreated with Vit, the elevated $\mathrm{Bax} / \mathrm{Bcl}-2$ ratio and caspase- 3 activity were both decreased significantly (Figure $3 \mathrm{E}-\mathrm{G},{ }^{*} P<0.05,{ }^{\sharp} P<0.05$ ).
These results implicated that Vit pretreatment protected SH-SY5Y cells against $\mathrm{MPP}^{+}$-induced toxicity and apoptosis.

\section{Vit suppresses $\mathrm{MPP}^{+}$-induced apoptosis via PI3K/Akt signaling pathway in $\mathrm{SH}-\mathrm{SY} 5 Y$ cells}

As shown in Figure 4, MPP ${ }^{+}$treatment downregulated the $\mathrm{p}-\mathrm{PI} 3 \mathrm{~K} / \mathrm{PI} 3 \mathrm{~K}$ ratio and $\mathrm{p}-\mathrm{Akt} / \mathrm{Akt}$ ratio while upregulated Bax/Bcl-2 ratio and caspase-3 activity in SH-SY5Y cells. When pretreated with Vit, the inhibitory effect of $\mathrm{MPP}^{+}$on the phosphorylation of PI3K and Akt and the promoting effect of $\mathrm{MPP}^{+}$on the apoptosis were abolished. To further confirm the anti-apoptotic effect of Vit mediated through PI3K/Akt signaling pathway, LY294002, an inhibitor for PI3K activation was employed. We found that in the presence of LY294002, Vit could not abolish the inhibitory 


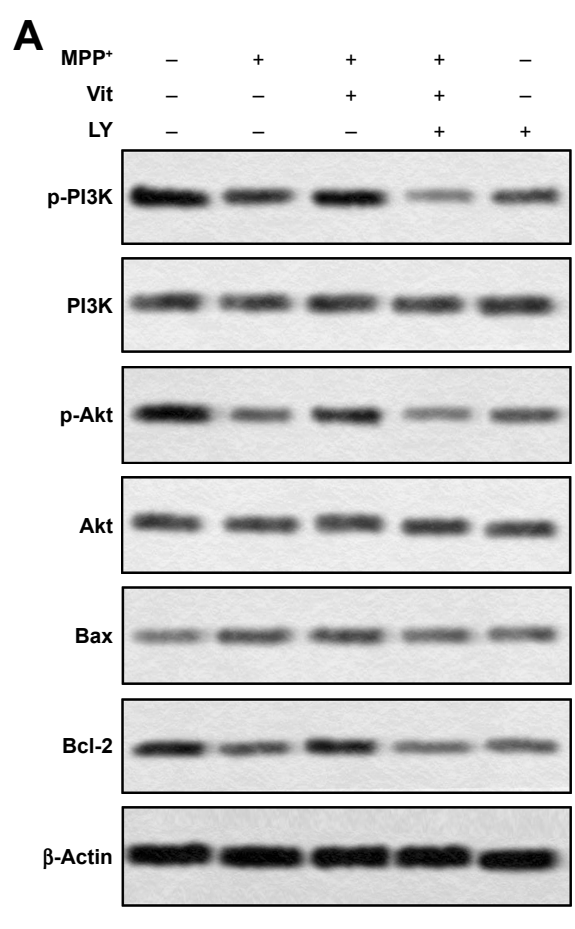

$\mathbf{F}$

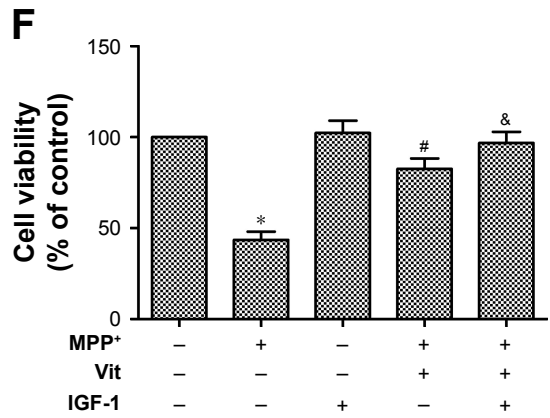

B

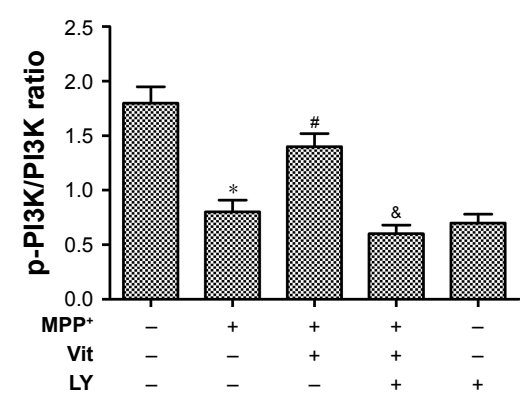

D

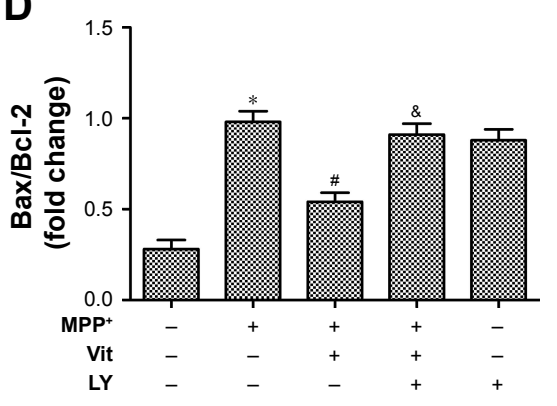

C

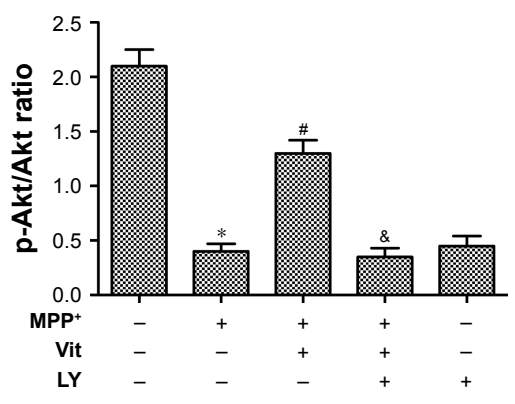

$E$

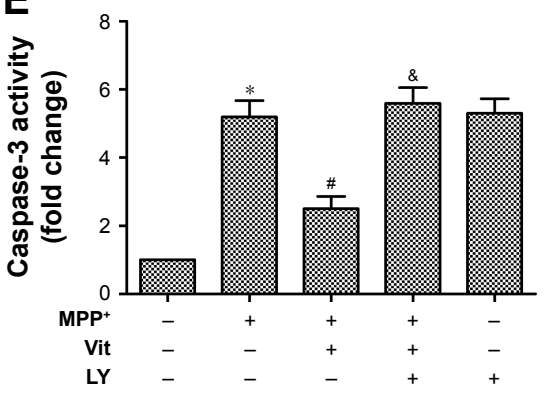

Figure 4 Vitexin suppresses MPP+-induced apoptosis via PI3K/Akt signaling pathway in SH-SY5Y cells. (A-D) Relative expression of p-PI3K, PI3K, p-Akt, Akt, Bax, and Bcl-2 in SH-SY5Y cells was detected through western blot. (E and $\mathbf{G})$ Caspase-3 activity was measured using Caspase-3 Activity Assay Kit. (F) Cell viability was detected through MTT. The bars represent mean \pm SD of three independent experiments. $* P<0.05$ compared with control group, ${ }^{*} P<0.05$ compared with $M P P^{+}$-treated group, ${ }^{\&} P<0.05$ compared with $\mathrm{MPP}^{+}+$Vit-treated group.

Abbreviations: Vit, vitexin; MPP, methyl-4-phenylpyridine.

effect of $\mathrm{MPP}^{+}$on the phosphorylation of PI3K and Akt in SH-SY5Y cells. Accordingly, the effect of Vit on the suppression of the activation of caspase-3 and enhancement of Bcl-2 was abolished in the presence of LY294002 (Figure 4A-E, ${ }^{*} P<0.05,{ }^{*} P<0.05,{ }^{\circledR} P<0.05$ ). We then used PI3K/Akt activator IGF-1 to treat SH-SY5Y cells. We observed that IGF-1 itself did not have significant effect on cell viability and caspase-3 activity. However, when cotreated with Vit, IGF-1 could enhance the promoted effect on cell viability and inhibitory effect on caspase-3 activity of Vit in $\mathrm{MPP}^{+}$-treated cells (Figure $4 \mathrm{~F}$ and $\mathrm{G}$, $\left.{ }^{*} P<0.05,{ }^{*} P<0.05,{ }^{s} P<0.05\right)$. Taken together, our data suggested that Vit suppressed $\mathrm{MPP}^{+}$-induced apoptosis via PI3K/Akt signaling pathway in SH-SY5Y cells.

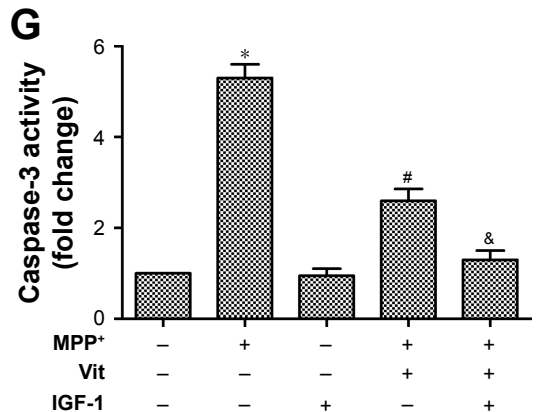

\section{Vit improves the lesioned neurobehavior of MPTP-treated mice}

Pole test and traction test were performed in our study to evaluate motor deficits and bradykinesia, respectively, in MPTP-treated mice. Results from the pole test indicated that MPTP treatment prolonged total locomotor activity time (Figure 5A, ${ }^{*} P<0.05,{ }^{\sharp} P<0.05$ ). Pretreatment with Vit shortened this time significantly, suggesting that Vit treatment prevented MPTP-induced bradykinesia. Results from traction test suggested that MPTP-treated mice showed decreased strength as their hind limb grip score was lower and the hanging time was also shorter than control group (Figure 5B and $\left.\mathrm{C},{ }^{*} P<0.05,{ }^{\#} P<0.05\right)$. However, pretreated with Vit increased traction score and hanging time, indicating that 

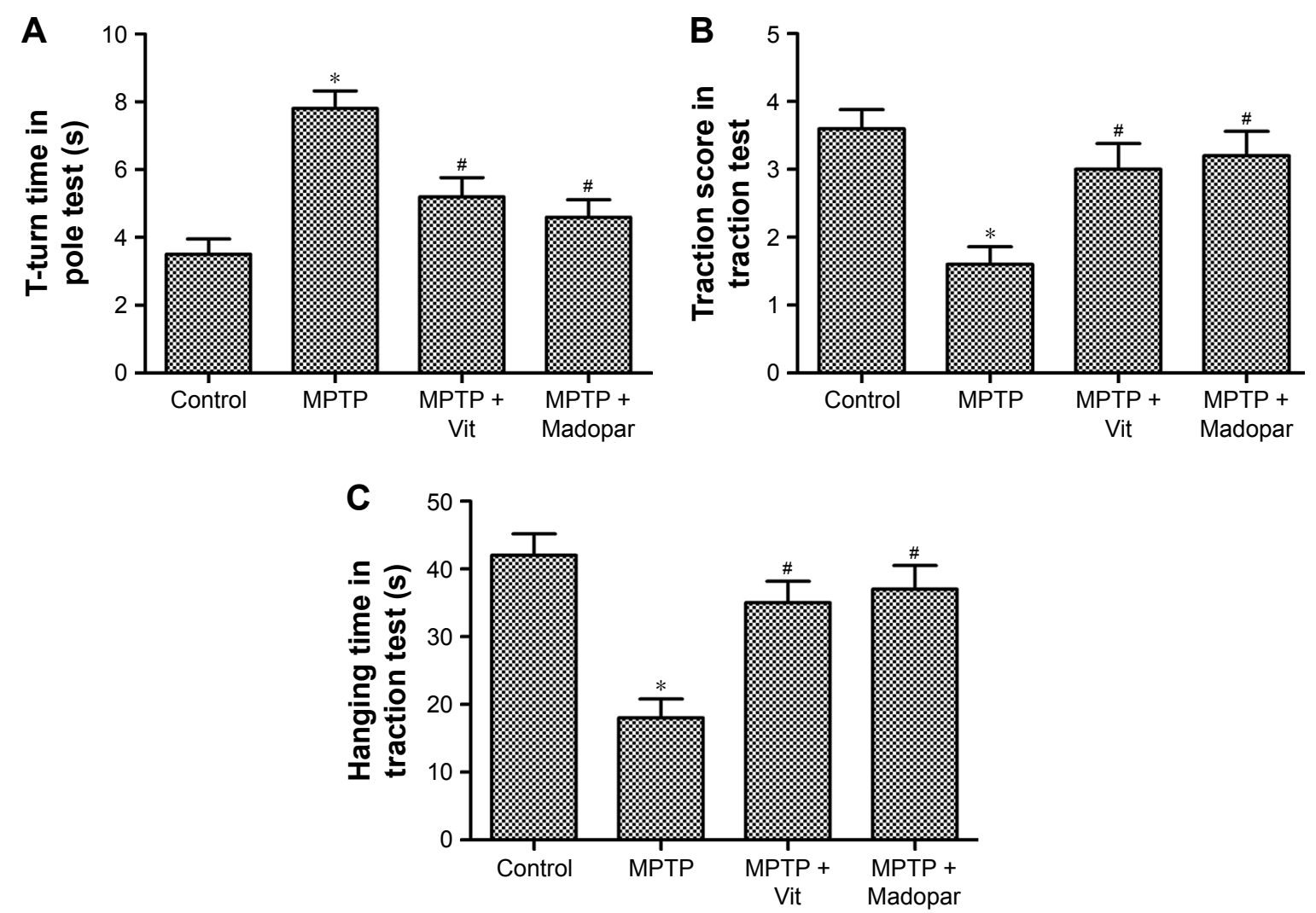

Figure 5 Vitexin improves the lesioned neurobehavior of MPTP-treated mice. The 32 mice were randomly divided into four groups with eight mice in each group: control group, MPTP group, MPTP + Vit group, MPTP + Madopar group. (A) T-turn time in the pole test. (B) Hind limb grip score in the traction test. (C) Hanging time in the traction test. $* P<0.05$ compared with control group, ${ }^{*} P<0.05$ compared with MPTP-treated group.

Abbreviations: Vit, vitexin; MPTP, I-methyl-4-phenyl-I,2,3,6-tetrahydropyridine.

prophylactic treatment with Vit could alleviate the initial lesions caused by MPTP (Figure 5B and C, $* P<0.05$, $\left.{ }^{\sharp} P<0.05\right)$. As a positive control drug, Madopar also decreased the T-turn time while increased traction score and hanging time in pole test and traction test significantly. These results suggested that Vit improved the lesioned neurobehavior of MPTP-treated mice.

\section{Vit attenuates apoptosis in MPTP-treated mice via PI3K/Akt signaling pathway}

Having known that Vit attenuated the apoptosis in SH-SY5Y cells, the in vivo effect of Vit on the apoptosis was also explored in our study. Our data showed that MPTP treatment decreased the $\mathrm{p}-\mathrm{PI} 3 \mathrm{~K} / \mathrm{PI} 3 \mathrm{~K}$ ratio and $\mathrm{p}-\mathrm{Akt} / \mathrm{Akt}$ ratio compared with control group, indicating that MPTP inhibited $\mathrm{PI} 3 \mathrm{~K} / \mathrm{Akt}$ signaling pathway in substantia nigra. Moreover, MPTP increased the Bax/Bcl-2 ratio and caspase-3 activity significantly. On the contrary, similar to Madopar, Vit treatment rescued the inhibited PI3K/Akt signaling pathway induced by MPTP and attenuated apoptosis by decreasing the $\mathrm{Bax} / \mathrm{Bcl}-2$ ratio and caspase-3 activity (Figure $6 \mathrm{~A}-\mathrm{G}$,
${ }^{*} P<0.05,{ }^{\#} P<0.05$ ). Our results indicated that Vit attenuated apoptosis in substantia nigra of MPTP-treated mice via $\mathrm{PI} 3 \mathrm{~K} /$ Akt signaling pathway.

\section{Discussion}

$\mathrm{PD}$ is a progressive neurodegenerative disease with many symptoms like bradykinesia, resting tremors, and rigidity. ${ }^{16,17}$ Flavonoid, which is widely present in vegetables, plants, and fruits, has been reported to exert a great number of neuroprotective effects. ${ }^{18}$ As one of the flavonoids, Vit has also been discovered to exert neuroprotective effect. However, whether Vit contributes to the alleviation of PD progression has not been elucidated before. In our present study, $\mathrm{MPP}^{+}$and MPTP were used to induce PD model in vitro and in vivo, respectively, and we tried to find out the effect of Vit on the treatment of PD. Our data indicated that Vit protected dopaminergic neurons in $\mathrm{MPP}^{+}$- or MPTP-induced PD through PI3K/Akt signaling pathway.

Since degeneration of dopaminergic neurons is the prominent characteristic of $\mathrm{PD}$, whether Vit could protect dopaminergic neurons in $\mathrm{MPP}^{+} / \mathrm{MPTP}-$ induced $\mathrm{PD}$ model 
A

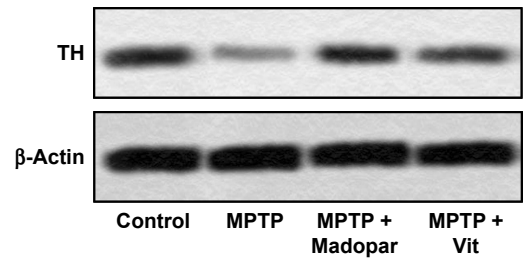

B

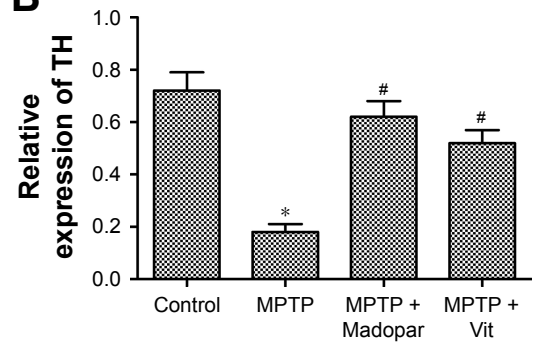

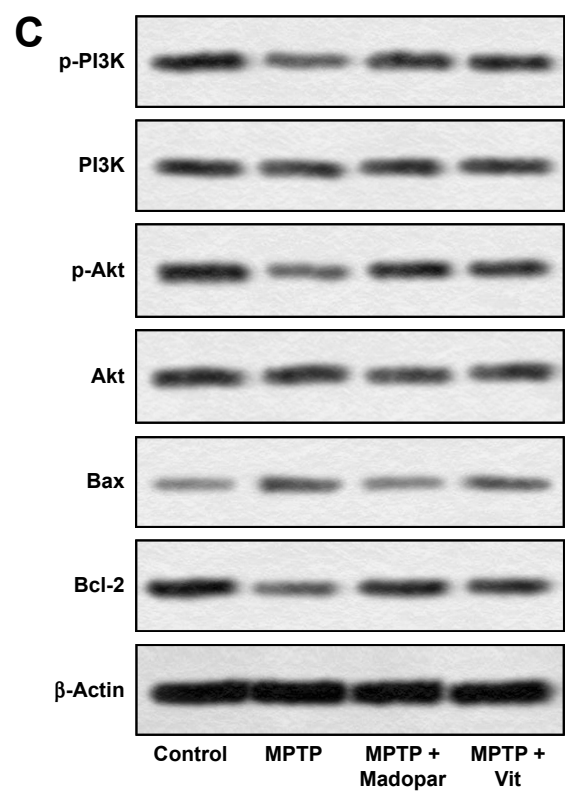

D

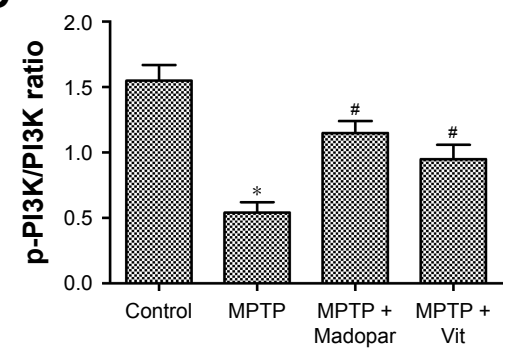

$\mathbf{F}$

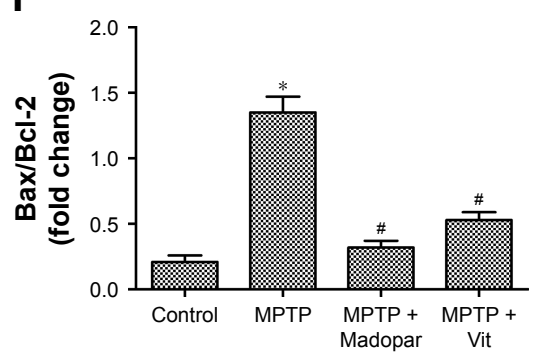

E

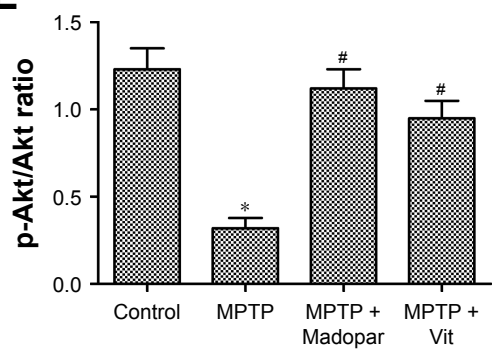

G

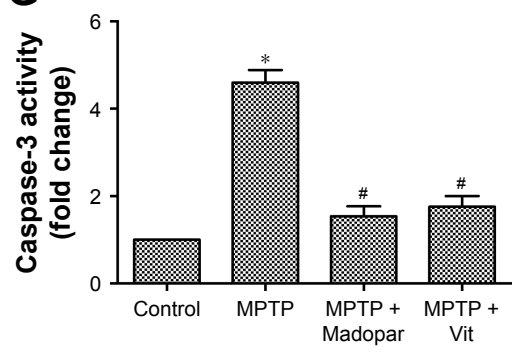

Figure 6 Vitexin attenuates apoptosis in MPTP-treated mice. The 32 mice were randomly divided into four groups with eight mice in each group: control group, MPTP group, MPTP + Vit group, MPTP + Madopar group. (A-F) Relative expression of p-PI3K, PI3K, p-Akt, Akt, Bax, and Bcl-2 in substantia nigra of mice was detected through Western blot. (G) Caspase-3 activity was measured using Caspase-3 Activity Assay Kit. The bars represent mean \pm SD of three independent experiments. $* P<0.05$ compared with control group, ${ }^{\sharp P}<0.05$ compared with MPTP-treated group.

Abbreviations: Vit, vitexin; MPTP, I-methyl-4-phenyl-1,2,3,6-tetrahydropyridine.

was first investigated in our study. The in vitro PD model on human SH-SY5Y cells was constructed under $\mathrm{MPP}^{+}$treatment as described before. ${ }^{19}$ Vit treatment upregulated the decreased cell viability while downregulated the increased cell apoptosis rate of SH-SY5Y cells induced by $\mathrm{MPP}^{+}$treatment significantly in a dose-dependent manner. The balance between pro-apoptotic protein Bax and anti-apoptotic protein Bcl-2 plays an important role in the regulation of apoptosis..$^{20}$ Caspase-3 is a class of cysteine proteases which is responsible for the proteolytic cleavage of many vital apoptosisrelated proteins, and cleaved caspase-3 is the hallmark for the activation of apoptosis..$^{21,22}$ Numerous studies indicated that $\mathrm{MPP}^{+}$or MPTP treatment disturbed the balance between Bax and Bcl-2 and also increased the activity of caspase- 3 in dopaminergic neurons. ${ }^{23-26}$ In agreement with these previous studies, we also observed that $\mathrm{MPP}^{+}$treatment increased the ratio of Bax/Bcl-2 and caspase-3 activity in SH-SY5Y cells. However, Vit treatment reversed these parameters induced by $\mathrm{MPP}^{+}$. Taken together, our results suggested that Vit protected $\mathrm{SH}-\mathrm{SY} 5 \mathrm{Y}$ cells against $\mathrm{MPP}^{+}$-induced toxicity and apoptosis. Zhou's study elucidated that Vit-induced apoptosis of breast cancer cells, which seemed to be a completely opposite effect from the neuroprotection effect demonstrated in our manuscript. However, the reason might be the different types of cells used in the experiments. Vit might have different effects on different cells. Moreover, Vit used in Zhou's study was isolated from the seed of Vitex Negundo while our Vit was purchased from company. Whether there was only Vit in the isolated compounds to induce apoptosis of breast cancer cells was largely unknown.

Flavonoids have been demonstrated to interact specifically with PI3K/Akt signaling pathways to exert their neuroprotective effects. ${ }^{10}$ Liu et al's study suggested that a flavonoid, Ginsenoside Rd protected SH-SY5Y cells against $\mathrm{MPP}^{+}$-induced injury via the PI3K/Akt survival signaling pathway. ${ }^{27}$ To explore the upstream signaling pathway 
involved in neuroprotective effect of Vit, the PI3K/Akt signaling pathway was investigated in our study. Similarly, we observed that Vit treatment attenuated the downregulation of phosphorylation of PI3K and Akt induced by $\mathrm{MPP}^{+}$. When PI3K pathway was blocked by LY294002, Vit could not reverse the high ratio of Bax/Bcl-2 and the high caspase-3 activity induced by $\mathrm{MPP}^{+}$treatment in SH-SY5Y cells. PI3K activator IGF-1 enhanced the effects of Vit. These results indicated that Vit suppressed $\mathrm{MPP}^{+}$-induced apoptosis through PI3K/Akt signaling pathway.

Having known the effect of Vit on the PD model in vitro, we then set to explore the role of Vit in the progression of $\mathrm{PD}$ in vivo. MPTP was used to treat male C57BL/6 mice for the construction of PD model in vivo because MPTP could cause PD-like symptoms such as bradykinesia and dopaminergic neuronal cell loss in SNpc. ${ }^{28}$ The pole test is a useful method to measure bradykinesia in mouse PD models, and the traction test is often used to evaluate muscle strength and equilibrium..$^{29,30}$ Madopar, the most widely used drug for PD therapy, contains two active ingredients, levodopa and benserazide, which contribute to the supplementation dopamine in the brain and was used as a positive control drug to assess the relative therapeutic efficacy of Vit in our study. ${ }^{31}$ Results from pole test and traction test showed that Vit improved the lesioned neurobehavior of MPTP-treated mice, indicating that Vit pretreatment prevented bradykinesia and alleviated the initial lesions caused by MPTP. Moreover, Vit treatment attenuated the downregulation of the phosphorylation of PI3K and Akt caused by MPTP and suppressed the ratio of $\mathrm{Bax} / \mathrm{Bcl}-2$ and caspase-3 activity significantly like Madopar did. Taken together, the data from in vivo experiments provided robust clues that Vit improved the lesioned neurobehavior and attenuated apoptosis in MPTP-treated mice through PI3K/Akt signaling pathway.

\section{Conclusion}

Our study demonstrated that Vit protected dopaminergic neurons against $\mathrm{MPP}^{+} / \mathrm{MPTP}$-induced neurotoxicity through the activation of PI3K/Akt signaling pathway. Our findings may facilitate the clinical application of Vit in the therapy of PD.

\section{Disclosure}

The authors report no conflicts of interest in this work.

\section{References}

1. Thomas B, Beal MF. Parkinson's disease. Hum Mol Genet. 2007; 16(Spec No. 2):R183-R194.

2. Mattson MP. Apoptosis in neurodegenerative disorders. Nat Rev Mol Cell Biol. 2000;1(2):120-129.
3. Onyango IG. Mitochondrial dysfunction and oxidative stress in Parkinson's disease. Neurochem Res. 2008;33(3):589-597.

4. Youn JK, Kim DW, Kim ST, et al. PEP-1-HO-1 prevents MPTP-induced degeneration of dopaminergic neurons in a Parkinson's disease mouse model. BMB Rep. 2014;47(10):569-574.

5. Utsumi H, Okuma Y, Kano O, et al. Evaluation of the efficacy of pramipexole for treating levodopa-induced dyskinesia in patients with Parkinson's disease. Intern Med. 2013;52(3):325-332.

6. Dauer W, Przedborski S. Parkinson's disease: mechanisms and models. Neuron. 2003;39(6):889-909.

7. Yi F, He X, Wang D. Lycopene protects against MPP(+)-induced cytotoxicity by maintaining mitochondrial function in SH-SY5Y cells. Neurochem Res. 2013;38(8):1747-1757.

8. Alcaraz-Zubeldia M, Rojas P, Boll C, Rios C. Neuroprotective effect of acute and chronic administration of copper (II) sulfate against MPP+ neurotoxicity in mice. Neurochem Res. 2001;26(1):59-64.

9. Vauzour D, Vafeiadou K, Rodriguez-Mateos A, Rendeiro C, Spencer JP. The neuroprotective potential of flavonoids: a multiplicity of effects. Genes Nutr. 2008;3(3-4):115-126.

10. Williams RJ, Spencer JP. Flavonoids, cognition, and dementia: actions, mechanisms, and potential therapeutic utility for Alzheimer disease. Free Radic Biol Med. 2012;52(1):35-45.

11. Wang Y, Zhen Y, Wu X, et al. Vitexin protects brain against ischemia/ reperfusion injury via modulating mitogen-activated protein kinase and apoptosis signaling in mice. Phytomedicine. 2015;22(3):379-384.

12. Prabhakar MC, Bano H, Kumar I, Shamsi MA, Khan MS. Pharmacological investigations on vitexin. Planta Med. 1981;43(4):396-403.

13. Nurdiana S, Goh YM, Ahmad H, et al. Changes in pancreatic histology, insulin secretion and oxidative status in diabetic rats following treatment with Ficus deltoidea and vitexin. BMC Complement Altern Med. 2017; 17(1):290.

14. Zhou Y, Liu YE, Cao J, et al. Vitexins, nature-derived lignan compounds, induce apoptosis and suppress tumor growth. Clin Cancer Res. 2009; 15(16):5161-5169.

15. Chen L, Zhang B, Shan S, Zhao X. Neuroprotective effects of vitexin against isoflurane-induced neurotoxicity by targeting the TRPV1 and NR2B signaling pathways. Mol Med Rep. 2016;14(6): 5607-5613.

16. Haga R, Sugimoto K, Nishijima H, et al. Clinical utility of skin biopsy in differentiating between Parkinson's disease and multiple system atrophy. Parkinsons Dis. 2015;2015:167038.

17. Liu HG, Zhang K, Yang AC, Zhang JG. Deep brain stimulation of the subthalamic and pedunculopontine nucleus in a patient with Parkinson's disease. J Korean Neurosurg Soc. 2015;57(4):303-306.

18. Solanki I, Parihar P, Parihar MS. Neurodegenerative diseases: from available treatments to prospective herbal therapy. Neurochem Int. 2016; 95:100-108.

19. Xie HR, Hu LS, Li GY. SH-SY5Y human neuroblastoma cell line: in vitro cell model of dopaminergic neurons in Parkinson's disease. Chinese Med J. 2010;123(8):1086-1092.

20. Cory S, Adams JM. The Bcl2 family: regulators of the cellular life-ordeath switch. Nat Rev Cancer. 2002;2(9):647-656.

21. Whelan RS, Kaplinskiy V, Kitsis RN. Cell death in the pathogenesis of heart disease: mechanisms and significance. Annu Rev Physiol. 2010; 72:19-44.

22. Tian HY, Li ZX, Li HY, Wang HJ, Zhu XW, Dou ZH. Effects of 14 single herbs on the induction of caspase- 3 in tumor cells: a brief review. Chin J Integr Med. 2013;19(8):636-640.

23. He H, Wang S, Tian J, et al. Protective effects of 2,3,5,4'tetrahydroxystilbene-2-O-beta-D-glucoside in the MPTP-induced mouse model of Parkinson's disease: Involvement of reactive oxygen species-mediated JNK, P38 and mitochondrial pathways. Eur $J$ Pharmacol. 2015;767:175-182.

24. Khwanraj K, Phruksaniyom C, Madlah S, Dharmasaroja P. Differential expression of tyrosine hydroxylase protein and apoptosis-related genes in differentiated and undifferentiated SH-SY5Y neuroblastoma cells treated with MPP(.). Neurol Res Int. 2015;2015:734703. 
25. Selvakumar GP, Manivasagam T, Rekha KR, Jayaraj RL, Elangovan N. Escin, a novel triterpene, mitigates chronic MPTP/p-induced dopaminergic toxicity by attenuating mitochondrial dysfunction, oxidative stress, and apoptosis. J Mol Neurosci. 2015;55(1):184-197.

26. Cao Q, Qin L, Huang F, et al. Amentoflavone protects dopaminergic neurons in MPTP-induced Parkinson's disease model mice through PI3K/Akt and ERK signaling pathways. Toxicol Appl Pharmacol.2017; 319:80-90.

27. Liu Y, Zhang RY, Zhao J, et al. Ginsenoside Rd protects SH-SY5Y cells against 1-methyl-4-phenylpyridinium induced injury. Int J Mol Sci. 2015;16(7):14395-14408.

28. Przedborski S, Jackson-Lewis V, Djaldetti R, et al. The parkinsonian toxin MPTP: action and mechanism. Restor Neurol Neurosci. 2000; 16(2):135-142.
29. Ju MS, Kim HG, Choi JG, et al. Cassiae semen, a seed of Cassia obtusifolia, has neuroprotective effects in Parkinson's disease models. Food Chem Toxicol. 2010;48(8-9):2037-2044.

30. Guo H, Shi F, Li M, Liu Q, Yu B, Hu L. Neuroprotective effects of Eucommia ulmoides Oliv. and its bioactive constituent work via ameliorating the ubiquitin-proteasome system. BMC Complement Altern Med. $2015 ; 15: 151$.

31. Wang JY, Yang JY, Wang F, et al. Neuroprotective effect of pseudoginsenoside-f11 on a rat model of Parkinson's disease induced by 6-hydroxydopamine. Evid Based Complement Altern Med. 2013;2013: 152798 .

\section{Publish your work in this journal}

Drug Design, Development and Therapy is an international, peerreviewed open-access journal that spans the spectrum of drug design and development through to clinical applications. Clinical outcomes, patient safety, and programs for the development and effective, safe, and sustained use of medicines are the features of the journal, which has also been accepted for indexing on PubMed Central. The manuscript management system is completely online and includes a very quick and fair peer-review system, which is all easy to use. Visit http://www.dovepress.com/testimonials.php to read real quotes from published authors.

Submit your manuscript here: http://www.dovepress.com/drug-design-development-and-therapy-journal 